\title{
Encoding of Vicarious Reward Prediction in Anterior Cingulate Cortex and Relationship with Trait Empathy
}

\author{
DPatricia. L. Lockwood, ${ }^{1}$ Matthew A.J. Apps, ${ }^{2,3}$ Jonathan P. Roiser, ${ }^{4 \star}$ and Essi Viding ${ }^{1 \star}$ \\ ${ }^{1}$ Division of Psychology and Language Sciences, University College London, London WC1H OAP, United Kingdom, ${ }^{2}$ Nuffield Department of Clinical \\ Neuroscience, University of Oxford, John Radcliffe Hospital, Oxford OX3 9DU, United Kingdom, ${ }^{3}$ Department of Experimental Psychology, University of \\ Oxford, Oxford OX1 3DU, United Kingdom, and 4Institute of Cognitive Neuroscience, University College London, London WC1N 3AR, United Kingdom
}

\begin{abstract}
Empathy - the capacity to understand and resonate with the experiences of others - can depend on the ability to predict when others are likely to receive rewards. However, although a plethora of research has examined the neural basis of predictions about the likelihood of receiving rewards ourselves, very little is known about the mechanisms that underpin variability in vicarious reward prediction. Human neuroimaging and nonhuman primate studies suggest that a subregion of the anterior cingulate cortex in the gyrus (ACCg) is engaged when others receive rewards. Does the ACCg show specialization for processing predictions about others' rewards and not one's own and does this specialization vary with empathic abilities? We examined hemodynamic responses in the human brain time-locked to cues that were predictive of a high or low probability of a reward either for the subject themselves or another person. We found that the ACCg robustly signaled the likelihood of a reward being delivered to another. In addition, ACCg response significantly covaried with trait emotion contagion, a necessary foundation for empathizing with other individuals. In individuals high in emotion contagion, the ACCg was specialized for processing others' rewards exclusively, but for those low in emotion contagion, this region also responded to information about the subject's own rewards. Our results are the first to show that the ACCg signals probabilistic predictions about rewards for other people and that the substantial individual variability in the degree to which the ACCg is specialized for processing others' rewards is related to trait empathy.
\end{abstract}

Key words: anterior cingulate; emotion contagion; empathy; fMRI; reward prediction; social reward

Significance Statement

Successfully cooperating, competing, or empathizing with others can depend on our ability to predict when others are going to get something rewarding. Although many studies have examined how the brain processes rewards we will get ourselves, very little is known about vicarious reward processing. Here, we show that a subregion of the anterior cingulate cortex in the gyrus (ACCg) shows a degree of specialization for processing others' versus one's own rewards. However, the degree to which the ACCg is specialized varies with people's ability to empathize with others. This new insight into how vicarious rewards are processed in the brain and vary with empathy may be key for understanding disorders of social behavior, including psychopathy and autism.

\section{Introduction}

The successful prediction of future rewards is fundamental for adaptive behavior. The neural mechanisms that underpin reward prediction for oneself are becoming increasingly well understood (Schultz, 2013). However, during social interactions, stimuli are

\footnotetext{
Received May 1, 2015; revised Aug. 18, 2015; accepted Aug. 23, 2015.

Author contributions: P.L.L., M.A.J.A., J.P.R., and E.V. designed research; P.L.L. performed research; P.L.L. analyzed data; P.L.L., M.A.J.A., J.P.R., and E.V. wrote the paper.

This work was supported by the Medical Research Council (doctoral training account studentship from the Medical Research Council to P.L.L.) and the Birkbeck-University College London Centre for Neuroimaging. M.A.J.A. is supported by an Anniversary Future Leaders fellowship from the Biotechnology and Biological Sciences Research Council (Grant BB/M013596/1). E.V. is supported by the Medical Research Council (Grant MR/K014080/1) and is a Royal Society Wolfson Research Merit Award holder. We thank Lewis Pollock and Jonathan Blott for acting as confederates and Philip Kelly, Elizabeth $0^{\prime}$ Nions, and Ana Seara-Cardoso for advice and help with data collection.

*J.P.R. and E.V. contributed equally to this work.
}

often predictors of rewards for others, not exclusively ourselves. Effectively cooperating, competing, or empathizing with another requires the ability to compute the value of stimuli that predict rewards for others (Ruff and Fehr, 2014). However, very little is known about how vicarious reward predictions are processed in the brain. Moreover, there is a dearth of knowledge regarding

\footnotetext{
This article is freely available online through the J Neurosci Author Open Choice option.

Correspondence should be addressed to Patricia L. Lockwood, Division of Psychology and Language Sciences, University College London, 26 Bedford Way, London WC1H 0AP, United Kingdom. E-mail: p.lockwood@ucl.ac.uk. DOI:10.1523/JNEUROSCI.1703-15.2015

Copyright $\odot 2015$ Lockwood et al.

This is an Open Access article distributed under the terms of the Creative Commons Attribution License Creative Commons Attribution 4.0 International, which permits unrestricted use, distribution and reproduction in any medium provided that the original work is properly attributed.
} 
how individual differences in social functioning are related to neural response to others' reward.

The dorsal anterior cingulate cortex (dACC) signals predictive information about reward value, including the probability and magnitude of future rewards (Shidara and Richmond, 2002; Rogers et al., 2004; Sallet et al., 2007). This region is also engaged when processing social information (Behrens et al., 2008; Lamm et al., 2011; Gabay et al., 2014). Recently, a model of the dACC was proposed that unifies these different facets of its function (Apps et al., 2013b). This model posits that a subregion of the ACC in the gyrus (ACCg), lying in the anterior portions of the midcingulate cortex (areas $24 \mathrm{a}^{\prime} / 24 \mathrm{~b}^{\prime}$; Vogt et al., 1995), is sensitive to processing information about rewards for other people, including probabilistic predictions about rewards that others are likely to receive (Apps et al., 2013b). Several lines of evidence support this model. First, there are neurons in the ACCg that respond when a monkey views cues indicating that another monkey will receive a reward (Chang et al., 2013) neurons and in the dACC that respond when monkeys predict the decisions of a conspecific in an economic game (Haroush and Williams, 2015). Second, lesions to the ACCg reduce the value assigned to social stimuli, leaving the processing of nonsocial stimuli intact (Rudebeck et al., 2006). Third, hemodynamic responses in this region vary with the net-value of rewards received by others, the volatility of social information, predictions about the value of others' actions, and predictions of social approval from others (Behrens et al., 2008; Jones et al., 2011; Boorman et al., 2013; Apps and Ramnani, 2014). Together, these studies point to a central role for the ACCg in processing information about others' rewards. However, a key untested hypothesis from this model is that the ACCg is engaged when predictions are made about the probability of another person receiving a reward. Therefore, the first aim of our study was to test this hypothesis.

A second hypothesis derived from this model is that individual differences in social functioning, specifically empathy, vary with the extent to which ACCg is specialized for processing others' rewards. Empathy can be broadly defined as the capacity to understand and resonate with the experiences of others (Singer and Lamm, 2009). Empirical and theoretical accounts have suggested that the ACC is involved in empathizing (Lamm et al., 2011; Engen and Singer, 2013), but prior work has largely focused on response of this region to processing others' pain and other negative outcomes (for review, see Lamm et al., 2011) rather than positive, rewarding outcomes. The propensity to feel empathy varies substantially between individuals (Blair, 2005; Lockwood et al., 2013a; Bird and Viding, 2014), but the mechanisms that underpin individual differences in vicariously processing another's rewards are poorly understood. Therefore, the second aim of our study was to test the hypothesis that the extent to which the ACCg is specialized for processing others' rewards is positively associated with trait empathy.

\section{Materials and Methods}

\section{Participants}

Thirty-two right-handed healthy males (age 19-32 years, $\mathrm{M}=22.7$, $\mathrm{SD}=3.0$ ) were recruited through university participant databases. Exclusion criteria included previous or current neurological or psychiatric disorder, non-normal or noncorrected to normal vision, non-native English language, and previous or current study of psychology. This latter criterion was used due to concerns that prior experience of studying psychology could compromise participants' belief in the deception used in the protocol. Two participants were excluded from the analysis (one due to excessive motion ( $>10 \%$ of scans) and one due to neurological abnormalities), leaving a final sample of 30 . All participants gave written informed consent and the study was approved by the local departmental research ethics committee.

\section{Experimental task}

Design. We examined the processing of cues that signaled the probability with which a first-person and a third-person would receive a reward. A $2 \times 2$ factorial design, agency (self vs other) and probability (high $80 \%$ vs low 20\%), was used to examine activation time-locked to the cues (see Fig. 1).

On each trial during the experiment, participants saw cues that indicated the probability with which they (first-person or "self") or the other participant (third-person or "other") were likely to win points. These cues were represented as pie charts to depict the level of probability explicitly and minimize any requirements for reward learning across the task. The cues for self and other differed in color, but were luminance matched. Self cues had the word "you" written above them, whereas other cues had the name of the other participant (a confederate) written above them. This ensured that participants were explicitly aware of whether the cues predicted outcomes for themselves or for the other participant.

After the cue, an outcome was presented. To ensure attention to the cues, participants indicated (at the time of the outcome) whether the outcome was expected or not with a button press. We specifically investigated passively delivered rather than instrumentally obtained rewards so that any activation differences between self and other trials could not be related to differences in motor preparation (e.g., an action on a self trial but no action on another trial).

Before scanning, participants completed a practice version of the task during which they received feedback as to whether their judgements (expected or unexpected) were correct. During scanning, however, participants were instructed that they would not receive feedback on their judgements, but that they should respond as quickly and accurately as possible to the judgment.

There were 100 trials in total, 50 self trials and 50 other trials presented in a pseudorandom order, with no more than three trials in a row of self or other cues. The 50 self trials consisted of 25 trials of high-probability first-person cues and 25 trials of low-probability first-person cues. Similarly, the 50 other trials consisted of 25 high-probability third-person cues and 25 low-probability first-person cues. For both the self and other conditions, 20 outcomes were an expected win, 20 outcomes were an expected no win, five outcomes were an unexpected win, and five outcomes were an unexpected no win (equivalent to $80 \% / 20 \%$ probability).

Trial structure. Each trial began with a cue that signaled the probability of reward (80\%/20\%) and agent (self/other) for $800 \mathrm{~ms}$ (see Fig. 1A). After a jittered delay (2500-6000 ms), participants observed an outcome (win 100 points/win 0 points; $800 \mathrm{~ms}$ ), followed by a variable fixation (2000-4000 ms). Participants were then presented with the options "yes" or "no" and were required to press one of the two buttons to indicate whether the outcome was expected or not. The side of the screen on which these options were presented was counterbalanced so that participants could not form a representation of a specific motor command at any point during a trial. Participants had $1500 \mathrm{~ms}$ to indicate their option or the word "missed" appeared in red on the screen. This was followed by a fixation cross (1000-2000 ms).

Procedure. Participants were paired with one of two age-matched confederates (who were also male), whom they believed were naive participants and had never met before the experiment. The participant and the confederate were instructed together that they could earn extra payment, based on the outcomes they received during the experimental task (see below); but in fact all participants were paid the same amount (total $£ 30$, representing an additional $\mathfrak{E} 7$ to the standard participant payment for the required time commitment). They also believed that the confederate participant could earn an extra payment in the same manner during the task. A set of standardized questions completed after the scan confirmed that no participant had become suspicious about the deception during the experiment.

Participants attended two sessions. The first session was attended only by the experimental participant without a confederate and involved practicing the experimental task and completing questionnaires. In the first 
session, attended only by the experimental participant, the "other" participant was described as "player 2" and the experimental participant was instructed that, in the scanning session, this name would be replaced by the name of the other participant. Participants were instructed that, during the practice session, the points would not be converted into any money either for themselves or the other person, but that when they attended the scanning session, these points would be converted into additional payment for themselves and the other participant. The second session ( $<7 \mathrm{~d}$ later) was attended by both the experimental participant and the confederate. During this session, the experimental participant performed the task while inside the MRI scanner. The experimental participant was under the impression that the confederate performed the same task simultaneously. The confederate was seated in the adjacent MRI control room to maintain this impression. The participants were instructed that, regardless of whether the cues and outcomes were for themselves or for the other person, they should perform the same judgment task to indicate whether the outcome was expected or not. Moreover, participants were not instructed to the specific payoff matrix, which was in fact equal. This was done to ensure that participants remained motivated to attend to the cues and outcomes.

After the scanning session, participants rated how positive they felt when observing themselves or the other person winning on a nine-point scale ranging from "not at all" to "very positive." One-sample $t$ tests showed that, for both self and other, participants felt significantly more positive than neutral when seeing win outcomes compared with no win outcomes (other win $t_{(29)}=2.1, p<0.05, \mathrm{M}=5.4, \mathrm{SD}=1.04$; self win $t_{(29)}=5.3 p<0.001, \mathrm{M}=6.4, \mathrm{SD}=1.43$ ), and paired-sample $t$ tests showed that participants felt significantly more positive when they won money for themselves compared with seeing the other participant win $\left(t_{(29)}=4.35, p<0.001\right)$. This suggests that participants found it rewarding to view win outcomes for both themselves and for the other participant, but felt more positive overall when they viewed themselves winning.

Questionnaire measures. Participants completed a measure of empathy, the Questionnaire of Cognitive and Affective Empathy (QCAE; Reniers et al., 2011). The QCAE is a multidimensional instrument devised to measure five key components of empathy. In the development of the QCAE, two raters selected items from other well validated and commonly used empathy measures (e.g., Empathy Quotient; Hogan Empathy Scale; the Empathy subscale of the Impulsiveness-Venturesomeness-Empathy Inventory; and the Interpersonal Reactivity Index) if they were deemed to measure empathy (see items below). Items deemed to measure other processes (e.g., sympathy) were not included. The five subscales comprising the QCAE are as follows: perspective-taking (e.g., "I can easily tell if someone else wants to enter a conversation."); online simulation (e.g., "Before criticizing somebody, I try to imagine how I would feel if I was in their place."); emotion contagion (e.g., "I am happy when I am with a cheerful group and sad when the others are glum."); peripheral responsivity (e.g., "I often get deeply involved with the feelings of a character in a film, play, or novel."); and proximal responsivity (e.g." "I often get emotionally involved with my friends' problems"). Items are rated on a four-point scale from "strongly disagree" to "strongly agree." The QCAE has good construct validity and internal consistency (Reniers et al., 2011).

Statistical analysis of behavioral data. Behavioral analyses were performed in SPSS 22 software (IBM). An agency (self vs other) by reward (win vs no win) ANOVA was used to examine reaction time (RT) differences to outcome judgments. We did not examine the agency (self vs other) by expectedness (expected vs unexpected) interaction due to the low number of unexpected outcomes in our design $(<10$ valid trials per subject). Relationships between behavioral performance and empathy were assessed using bivariate correlations. We adopted an $\alpha$ level of 0.05 and a power analysis indicated that we had $\sim 80 \%$ power to detect an effect size of Cohen's $d=0.50$.

\section{Functional neuroimaging data collection and analysis}

fMRI data acquisition. A Siemens Avanto 1.5-T MRI scanner was used to acquire a $5.5 \mathrm{~min} 3 \mathrm{D}$ T1-weighted structural scan and 424 multislice $\mathrm{T} 2 *$-weighted echoplanar volumes with BOLD contrast. The structural scan was acquired using a magnetization prepared rapid gradient echo sequence. Imaging parameters were as follows: 176 slices; slice thickness $=1 \mathrm{~mm}$; gap between slices $=0.5 \mathrm{~mm} ; \mathrm{TR}=2730 \mathrm{~ms} ; \mathrm{TE}=3.57$ $\mathrm{ms}$; field of view $=256 \mathrm{~mm} \times 256 \mathrm{~mm}^{2}$; matrix size $=256 \times 256$; voxel size $=1 \times 1 \times 1 \mathrm{~mm}$ resolution. The echoplanar image $(\mathrm{EPI})$ sequence was acquired in an ascending manner, at an oblique angle $\left(\approx 30^{\circ}\right)$ to the AC-PC line to decrease the impact of susceptibility artifact in the orbitofrontal cortex (Deichmann et al., 2003) with the following acquisition parameters: $424 \mathrm{~T} 2{ }^{\star}$-weighted echoplanar volumes, $352 \mathrm{~mm}$ slices, 1 $\mathrm{mm}$ slice gap; echo time $=50 \mathrm{~ms}$; repetition time $=2975 \mathrm{~ms}$; flip angle $=$ $90^{\circ}$; field of view $=192 \mathrm{~mm}$; matrix size $=64 \times 64$.

fMRI data analysis. Imaging data were analyzed using SPM8 (www.fil. ion.ucl.ac.uk/spm). Data preprocessing followed a standard sequence. The first four volumes were discarded to allow for $\mathrm{T} 1$ equilibration effects and the last volume was discarded because the experimental task ended one volume before the end of the scanning sequence. The removal of the last volume ensured that no hemodynamic response (which typically occurs 4-6 s after event onset) to the desktop screen was sampled. Images were then realigned and coregistered to the participant's own anatomical image. The anatomical image was processed using a unified segmentation procedure combining segmentation, bias correction, and spatial normalization to the Montreal Neurological Institute (MNI) template using SPM's New Segment procedure (Ashburner and Friston, 2005); the same normalization parameters were then used to normalize the EPI images. The images were resampled to a voxel size of $1.5 \times 1.5 \times$ $1.5 \mathrm{~mm}$. Finally, a Gaussian kernel of $8 \mathrm{~mm}$ full-width at half-maximum was applied to spatially smooth the images. Before the study, first-level design matrices were examined to ensure that estimable GLMs could be performed with independence between all regressors with correlation coefficients of $r<0.25$.

First-level analysis. Nine (in some subjects, 10) event types were used to construct regressors in which event onsets were convolved with the synthetic canonical hemodynamic response function in SPM and associated responses were estimated in the context of the general linear model. Each of the four conditions (self high probability, self low probability, other high probability, other low probability) at the time of the cue and at the time of the outcome was modeled as a separate regressor for correct responses. The onset of the judgment was also modeled in a single regressor across all event types. An additional regressor modeled trials in which the judgment was missed or participants made an error. For those participants whose head motion caused visible image corruption in particular scans, an extra regressor was included. These images were removed and replaced with an image created by interpolating the two adjacent images to prevent distortion of the between-subjects mask (four participants, each accounting for $<1 \%$ of the total fMRI data). The residual effects of head motion were also modeled as covariates of no interest in the analysis by including the six head motion parameters estimated during realignment. Data were high-pass filtered at $128 \mathrm{~s}$ to remove low-frequency drifts and the statistical model included an AR(1) autoregressive function to account for autocorrelations intrinsic to the fMRI time series. Contrast images were computed to examine the interaction (agency $\times$ probability) and main effects of agency (self $>$ other and other $>$ self) and probability (high $>$ low and low $>$ high) at the time of the cue.

Many studies have suggested that situations that involve mixed payoffs between study participants and other people can result in neural responses that reflect payoff differences between self and other; that is, they relate to coding of rewards for self relative for other, often called "inequity aversion" rather than "vicarious" reward responses (for reviews, see Ruff and Fehr, 2014; Rilling and Sanfey, 2011). To determine whether identified neural responses to reward predicting cues in the current study were reflective of coding of rewards for self relative to other, and thus inequity aversion, we constructed a second model that was the same as the main model but contained all cues collapsed into a single regressor. This regressor had two associated parametric modulators. The first coded the "inequity," the difference in accumulated reward between self and other on each trial, and the second coded the agent $\times$ probability interaction. This allowed us to determine neural responses to inequity and whether any neural responses occurred over and above the variance explained by inequity. 

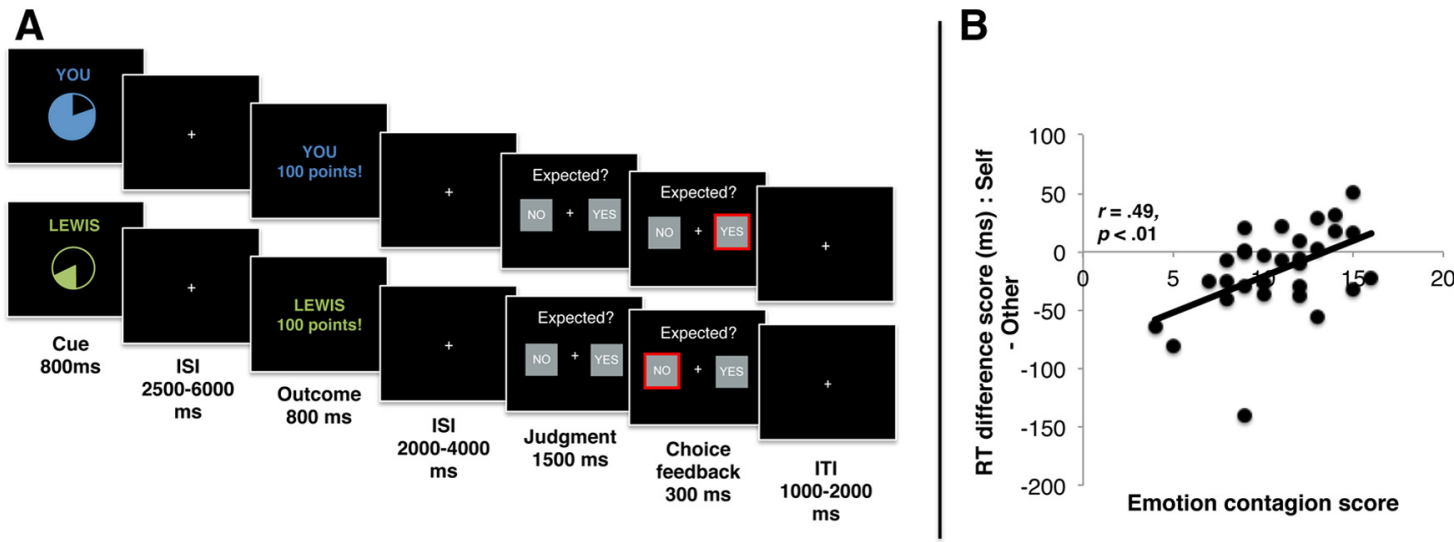

Figure 1. A, Trial structure. Participants performed trials that began with a cue signaling the probability of reward (high [80\%] or low [20\%]) and the agent to whom reward would be delivered (self = "you" and other = "Lewis" in this example). Participants judged whether the outcome (win 100 points or win 0 points) was expected or unexpected after outcome delivery. Participants believed that the other participant outside of the scanner was simultaneously performing the same task and that the points they observed would be converted into additional payment at the end of the experiment for themselves and for the other participant. $\boldsymbol{B}$, Scatterplot showing association between self-other RT difference at the time of the judgment and trait emotion contagion $(n=30)$. Overall, participants were slower when making judgments about the expectedness of outcomes for other compared with self. However, this effect was associated with emotion contagion such that those highest in emotion contagion showed a relative speeding of response for other.

Second-level analysis. Second-level analysis was performed using the standard summary statistics approach to random-effects analysis in SPM. Contrast images were input into second-level one-sample $t$ test design matrices. Interactions and main effects are reported at $p<0.05$, familywise error (FWE) corrected at the voxel level across the whole brain. Where significant interactions were identified, we conducted illustrative post hoc analyses with simple main effects contrasts using a less conservative statistical threshold of $p<0.001$ (uncorrected).

\section{Results}

\section{Behavioral data}

Participants were highly accurate in their judgments of whether the outcome was expected or not (mean accuracy $>91 \%$ for all participants for both trial types) and missed very few trials (mean $<1 \%$ for all participants). For mean RTs, a 2 (self vs other) by 2 (win vs no win) ANOVA showed significantly slower judgments on third-person $(\mathrm{M}=664 \mathrm{~ms}, \mathrm{SD}=18)$ than on first-person $(\mathrm{M}=649 \mathrm{~ms}, \mathrm{SD}=16)$ trials (main effect of agency: $F_{(1,29)}=$ 5.32, $p=0.03)$. Judgments were also significantly faster after reward $(641 \mathrm{~ms}, \mathrm{SD}=16)$ compared with a no reward $(672 \mathrm{~ms}$, $\mathrm{SD}=19$ ) outcomes (main effect of outcome $F_{(1,29)}=14.34, p=$ $0.001)$. The agency $\times$ reward interaction was nonsignificant $\left(F_{(1,29)}=.05, p=0.83\right)$.

Given the significant main effect of agency, we calculated the difference score between self and other RTs to examine associations between this behavioral measure and empathy. The emotion contagion subscale of the QCAE was positively associated with the self-other difference score $(r=0.49, p<0.01)$; that is, participants higher in emotion contagion showed a relative facilitation (speeding) when making decisions about the expectedness of outcomes for other people (Fig. 1B). No other subscale of the QCAE correlated with the self-other difference score (all $p>$ 0.49). Multiple regression, including all QCAE subscales, showed that the association between the self-other difference score and self-reported empathy was specific to the emotion contagion subscale $(\beta=0.55$, SEM $=2.43, p<0.01)$.

\section{fMRI data}

Agency $\times$ probability interaction at time of the cue

To test our first hypothesis, that activity in the ACCg would signal information about reward probability for others, we examined the agency $\times$ probability interaction at the time of the cue. Con- sistent with our hypothesis, this analysis revealed a significant effect in the ACCg (MNI coordinates $[x=8, y=32, z=12], Z=$ $5.05, k=10, p<0.05$ FWE, whole brain corrected), putatively in area $24 \mathrm{a}^{\prime} / 24 \mathrm{~b}^{\prime}$ at the border of the midcingulate and anterior cingulate subregions (Fig. 2). We examined the nature of this interaction by testing the simple main effects, specifically the contrasts of other high versus low probability and self low versus high probability. Inspection of the other high versus low probability simple main effect revealed a large cluster in the ACCg overlapping with the region identified in the interaction (MNI coordinates $[x=6, y=33, z=12], Z=4.14, k=184, p<0.001$, uncorrected). Inspection of the self low versus high probability contrast revealed a small cluster of overlapping voxels (MNI coordinates $[x=9, y=32, z=13], Z=3.28, k=5, p<0.001$, uncorrected). This suggests that the ACCg activation identified in the interaction mainly signals the probability of rewards that would be received by another person.

\section{Associations with trait empathy}

To test our second hypothesis, that the extent to which ACCg responds to the probability of rewards specifically for others would be positively associated with trait empathy, we used Mars$\mathrm{BaR}$ (Brett et al., 2002) to extract individual interaction contrast estimates (other high vs low probability minus self high vs low probability) from the ACCg cluster identified above and correlated these with participants' self-reported empathy on the five QCAE subscales. Emotion contagion was significantly negatively associated with the ACCg interaction contrast estimate $(r=-0.45, p=0.01$, all other subscales $p>0.58)$ and multiple regression, including all QCAE subscales, showed that this effect was specific to emotion contagion $(\beta=-.60$, SEM $=0.062, p=$ 0.003 , all other subscales $p>0.15$; Fig. 3 ). In other words, the interaction was weakest in individuals high in emotion contagion.

To better understand the nature of this association, we examined the correlations for other high versus low probability and self low versus high probability in ACCg with empathy subscales (Fig. 3). There was no significant correlation between ACCg response to other high versus low probability $(r=-0.05, p=0.81)$ and empathy. However, there was a significant negative association between ACCg response to self low versus high probability and emotion contagion $(r=-0.58, p<0.001)$; again, multiple 
A

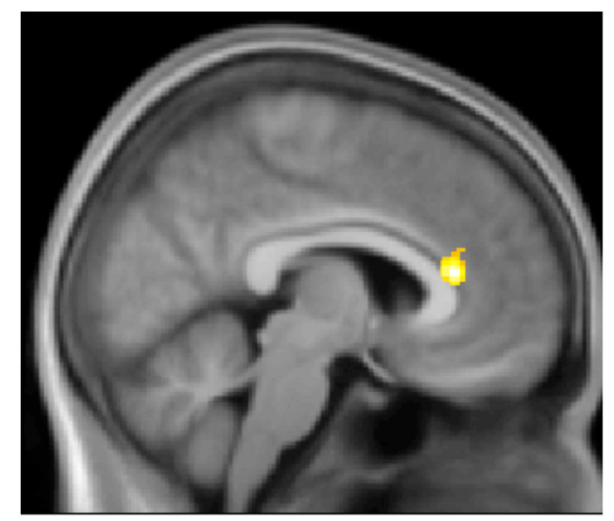

B

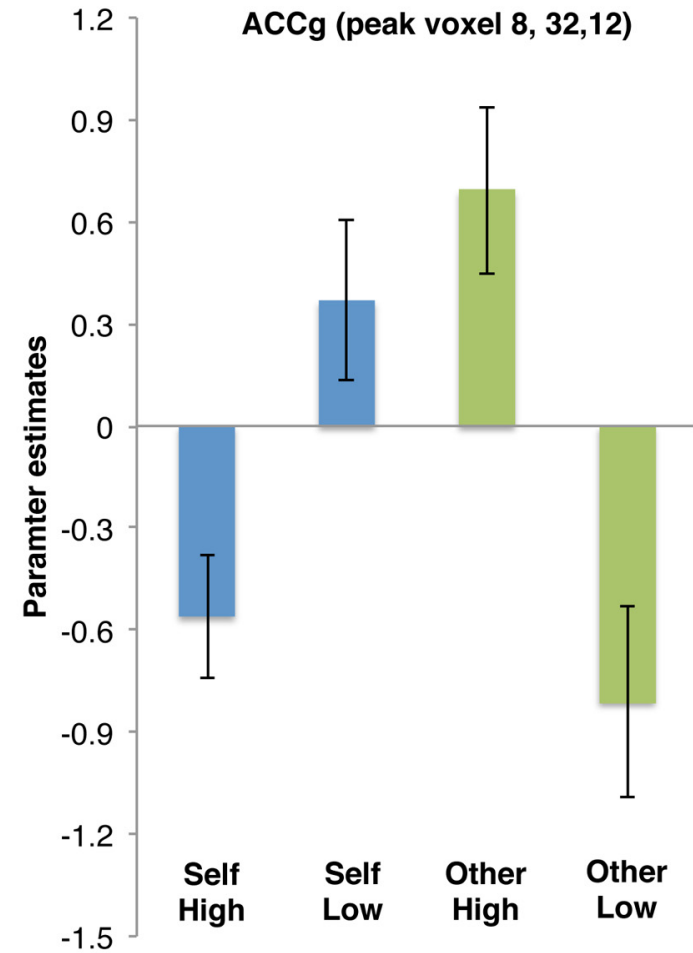

\section{Interaction Self low $>$ high Other high $>$ low}

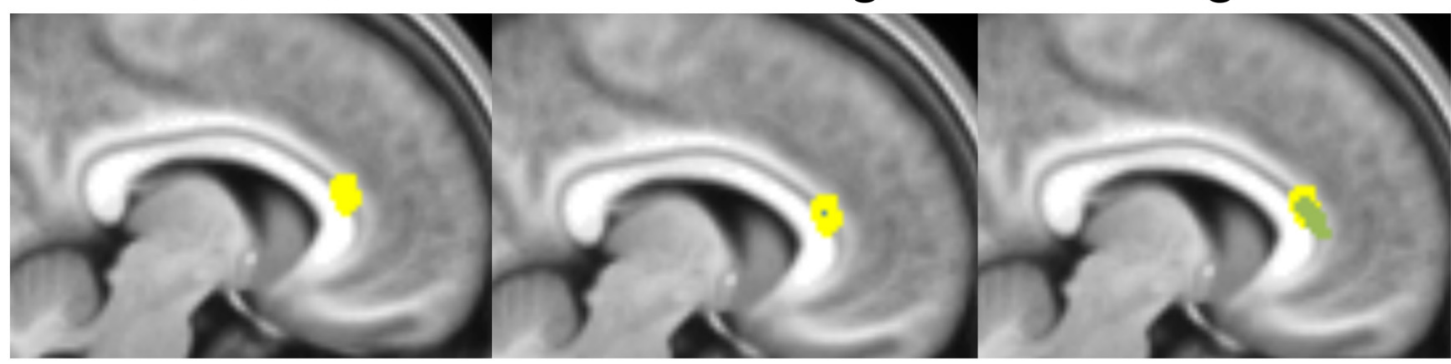

Figure 2. A, Activation in the ACCg signaled the agency (self vs other) by probability (high [80\%] or low [20\%]) interaction at the time of the cue $[x=8, y=32, z=12]$, displayed at $p<0.001$ (uncorrected). $\boldsymbol{B}$, Parameter estimates for the peak voxel in the ACC. C, Left, Overlay of the agency $\times$ probability interaction in ACCg (yellow, as in $\boldsymbol{A}$ ). Middle, Only a small number of voxels overlapped between the interaction contrast (yellow) and the simple main effect of self low versus high probability (blue, $k=5$ at $p<0.001$ uncorrected). Right, A large number of voxels overlapped between the interaction contrast (yellow) and the simple main effect of other high $>$ low probability (green, $k=184$ at $p<0.001$ uncorrected). Error bars indicate SEM.

regression demonstrated that this effect was unique to emotion contagion $(\beta=-0.66 \mathrm{SEM}=0.082, p<0.001$, all other subscales $p>0.19)$. In other words, the extent to which ACCg distinguished between low and high reward probability for self was attenuated in individuals with high emotion contagion.

In summary, in individuals with high emotion contagion, the ACCg signaled information about the relative difference between high and low probability rewards only for others, whereas in individuals with low emotion contagion, the ACCg additionally signaled (negatively) reward probability for self.

Main effects at the time of the cue

The temporal pole showed a significant main effect of other $>$ self (MNI coordinates [33, 22, -26$] ; Z=4.85 ; k=2, p<0.05$, FWE whole brain corrected). No other main effects or interactions survived whole-brain correction for multiple comparisons.

Agency $\times$ outcome interaction and main effects at the time of the outcome

No interactions or main effects survived whole-brain correction for multiple comparisons.

\section{Analysis of inequity aversion}

Analysis of the inequity parametric modulator showed no wholebrain-corrected results and no uncorrected results in ACCg. We then tested whether our observed effects in the ACCg occurred over and above any effects of inequity. This analysis showed that there was still a significant effect in the ACCg after accounting for the variance explained by inequity (MNI coordinates $[x=6, y=$ $32, z=13$ ] $, Z=4.97, k=8, p<0.05$ FWE, whole brain corrected). Therefore, the ACCg response was unlikely to reflect differences in accumulated reward between self and other.

\section{Discussion}

We examined hemodynamic responses in the human brain to cues that predicted a high or low probability of a reward for oneself or another person. We show that the ACCg robustly signals the probability of rewards for another person. This supports our hypothesis that the ACCg is engaged when processing predictions about rewards for other people. Our second hypothesis, that that the extent to which the ACCg is specialized for processing others' rewards is positively associated with trait empathy, 

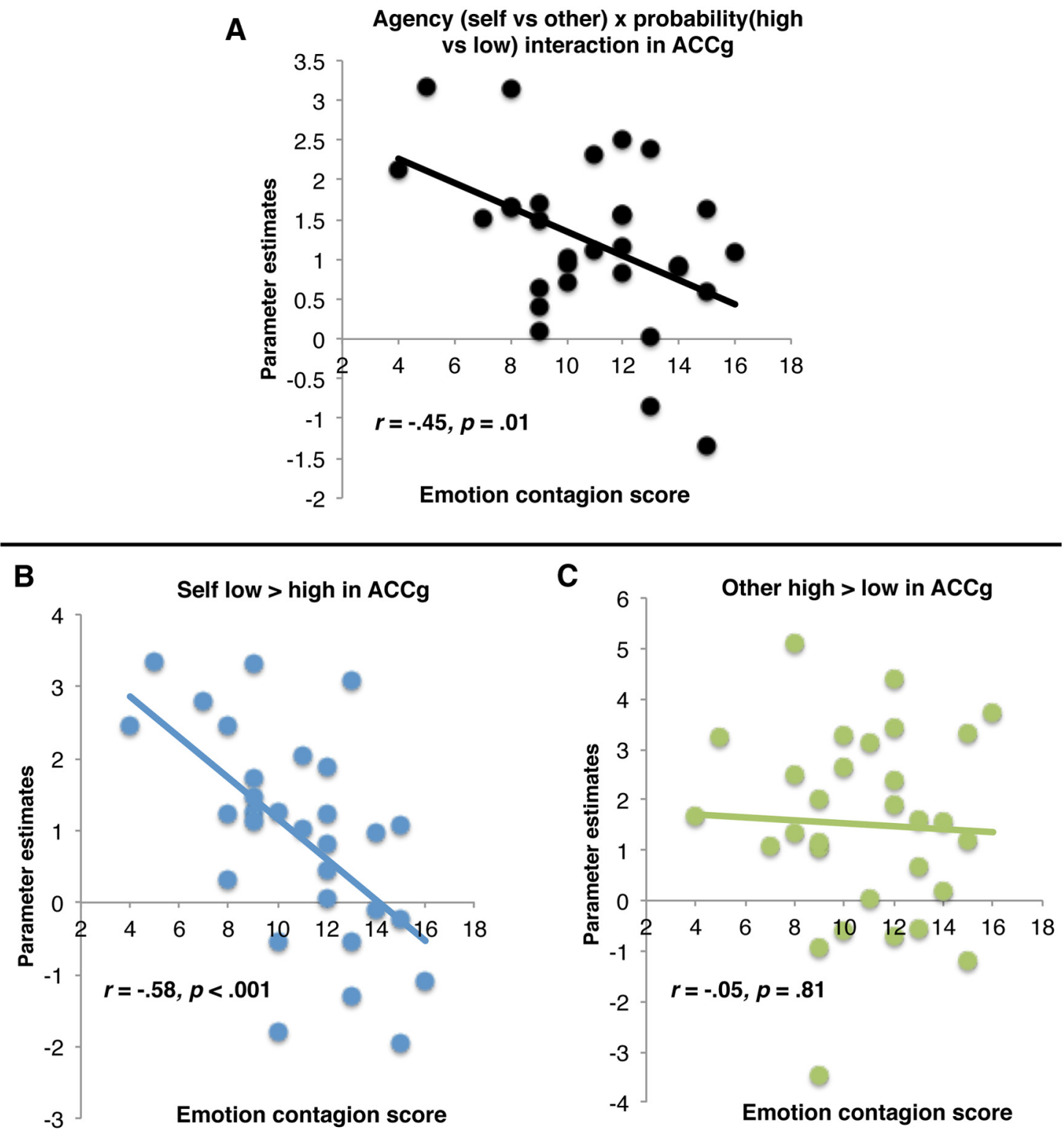

Figure 3. A, Significant association between the cluster in the ACCg showing the interaction effect and participants' emotion contagion scores. $\boldsymbol{B}$, Response to self low $>$ high probability decreases as a function of emotion contagion, with those lowest in emotion contagion showing the greatest response to low $>$ high probability of reward for self. $\boldsymbol{C}$, Response to other high $>$ low probability shows no significant modulation as a function of emotion contagion.

was partially supported. As predicted, the interaction effect in the ACCg significantly covaried with emotion contagion. However, this effect was driven by the extent to which ACCg signaled reward predictions for self, not other. Specifically, for those high in emotion contagion, the ACCg signaled reward prediction exclusively for others, whereas for those low in emotion contagion, this same region signaled reward prediction for self (in the opposite direction).

The model of the contributions of ACCg to social cognition (Apps et al., 2013b) highlights that this region plays an important role in understanding the value of others' rewards, and consequently in social behavior (Rudebeck et al., 2006; Behrens et al., 2008; Jones et al., 2011; Apps et al., 2013a, 2015; Boorman et al., 2013; Chang et al., 2013; Apps and Ramnani, 2014). This claim is built upon several lines of evidence. Lesions to this region have been shown to impair the processing of social stimuli and cause a reduction in the execution of social behaviors (Rudebeck et al.,
2006). The ACCg is connected to regions that process social information, but also to regions that process reward-related information (Yeterian and Pandya, 1991; Lynd-Balta and Haber, 1994; Haber et al., 1995). Single-unit recording evidence suggests that a relatively large proportion of ACCg neurons, compared with those in other prefrontal regions, respond when a monkey anticipates the delivery of reward to another monkey (Chang et al., 2013), and human imaging studies have shown that the ACCg responds when tracking the value of cues predicting approval from peers (Jones et al., 2011). Together, these studies support the claim that the ACCg is important for processing others' rewards and also in social behavior. However, a key untested component of this model was that the ACCg would be engaged when processing the likelihood of rewards being delivered to others. We show for the first time that the ACCg signals the likelihood of others' rewards regardless of trait levels of empathy. We also note that we did not observe responses to reward prediction in other 
candidate regions for reward signals, even at uncorrected levels (e.g., ventral striatum, ventromedial prefrontal cortex, and amygdala; for a meta-analysis, see Morelli et al., 2015), supporting some degree of specificity of ACCg response to vicarious reward in our study.

Our experimental paradigm was designed to ensure that participants attended to reward cues. By asking participants to make a decision at the time of the outcome, we cannot purely assess whether outcome-related responses are also coded in ACCg because participants were both processing the outcome and preparing a motor response during this time. However, there is evidence that vicarious prediction error signals may well be coded in ACCg (Apps et al., 2013a, 2015). We provide the first evidence that this same region also encodes the likelihood of others receiving rewards.

Although previous studies have suggested the ACCg plays an important role in empathy (Lamm et al., 2011; Engen and Singer, 2013), these studies have largely focused on neural responses to others' pain. Our data suggest that the degree of specialization in this region's response to others' predicted rewards may partly underlie individual differences in emotion contagion. Emotion contagion is hypothesized to be a necessary foundation for empathizing with other individuals (Bird and Viding, 2014) and is a process that is shared with nonhuman animals (for review, see de Waal, 2008). Importantly, emotion contagion also covaried with RTs to decisions about rewards delivered to others, with those highest in trait emotion contagion showing the greatest speeding of response. A distinction is often made between "affective empathy," which is commonly understood as an affective state caused by vicariously processing the experiences of another person, and "cognitive empathy," which is thought to include processes such as perspective taking and theory of mind (Singer and Lamm, 2009). Regression analyses suggested that only emotion contagion, part of the "affective" component, was associated with vicarious reward prediction. In tasks investigating cognitive aspects of empathy, an anatomically separate region of the $\mathrm{mPFC}$, the dorsal $\mathrm{mPFC}$, is often responsive (Amodio and Frith, 2006), suggesting partially separate functions of the ACCg and mPFC.

Although we did not predict an association between emotion contagion and ACCg response to self reward prediction, a possible explanation relates to the findings of Chang et al. (2013) and Haroush and Williams (2015). These investigators observed some self-reward- selective neurons in the same region of the ACCg/dACC that also contained other-reward-selective neurons, suggesting that some processing of information about rewards for self occurs in ACCg. However, given the limited sample sizes in nonhuman primate studies, the investigators were unable to examine variability in the proportion of neurons that signaled self versus other reward. We speculate that, even if at the population level, the ACCg shows a relative specialization in processing rewards for others, individual variability in the degree to which self rewards are also processed in this region could be important for explaining heterogeneity in ACCg function and empathy. That is, for those individuals who display the lowest levels of emotion contagion, there appears to be reduced specialization and a potentially opposing coding scheme of self and other reward probability in ACCg. Such opposing coding within the same anatomical region could have consequences for understanding social cognition and behavior, such as increased weighting of rewards to self and higher likelihood of engaging in competitive social interactions.
This interpretation is supported by a recent study finding that stimulation of dACC neurons made monkeys more competitive (Haroush and Williams, 2015). Similarly, another study showed that single neurons in a region of the rat cingulate cortex thought to be homologous with human dACC coded the value of competing with another rat for rewards (Hillman and Bilkey, 2012). These findings may help to reconcile previous discrepancies in the functions that have been imputed to dACC in terms of competitive social behaviors (Hillman and Bilkey, 2012; Haroush and Williams, 2015), but also empathy (Lamm et al., 2011; Engen and Singer, 2013). We propose that variability in empathy may modulate, not only the extent to which social information is processed in ACCg, as suggested in previous studies and theoretical accounts of empathy (Lamm et al., 2011; Engen and Singer, 2013), but also the extent to which self and other reward information is computed. However, this hypothesis requires further testing in future experiments.

Empathic abilities are a fundamental building block for successful social behavior and are at the core of many disorders of social cognition, including autism and psychopathy (Blair, 2005; Lockwood et al., 2013a; Bird and Viding, 2014). Previous studies have suggested that a similar portion of the dACC that was activated in our study is anatomically and functionally atypical in individuals with psychopathy and in individuals with autism (Simms et al., 2009; Brazil et al., 2011; Anderson and Kiehl, 2012; Delmonte et al., 2013; Lockwood et al., 2013b). Integrating these previous findings with the present results suggests the hypothesis that individual differences in the structure, function, and connectivity of the ACCg constrain the extent to which this region processes reward-predicting cues for others compared with self, which may lead to atypical empathic processing. However, we also know that psychopathy and autism have different profiles of empathic processing and behavior from one another (Blair, 2005; Lockwood et al., 2013a; Bird and Viding, 2014). The ACCg has strong connections to other regions involved in social and reward processing, including the nucleus accumbens (Yeterian and Pandya, 1991; Lynd-Balta and Haber, 1994; Haber et al., 1995), a region also suggested to participate in vicarious reward processing (Mobbs et al., 2009; Fareri et al., 2012; Braams et al., 2014), the temporal poles (which showed greater response to other vs self reward prediction in our study), and the temporoparietal junction and paracingulate cortex (Markowitsch et al., 1985; Seltzer and Pandya, 1989; Barbas et al., 1999). Future research into the neurocognitive correlates of psychopathy and autism should investigate whether distinct social behavioral abnormalities can be characterized by differences in the functional and connectional fingerprint of the ACCg during vicarious reward processing

In summary, we demonstrate a central role for the ACCg in processing predictions about the likelihood of others' rewards. We also found substantial individual variation in the degree to which the ACCg responds to self and other reward, with only those highest in trait emotion contagion showing specialization of ACCg for others predicted reward. Together, our findings highlight the importance of understanding the contributions of the ACCg to social cognition and how variability in its function may underlie variability in social behavior.

\section{Notes}

Supplemental material for this article is available at http://www. patricialockwood.co.uk/Publications. In Table 1, we provide uncorrected results at the time of the cue $(p<0.001, k=10)$ for completeness. We note that these results should be interpreted with caution given that they do not survive correction for multiple comparisons. In Table 2, we 
provide uncorrected results at the time of the outcome $(p<0.001, k=$ 10) for completeness. We note that these results should be interpreted with caution given that they do not survive correction for multiple comparisons. This material has not been peer reviewed.

\section{References}

Amodio DM, Frith CD (2006) Meeting of minds: the medial frontal cortex and social cognition. Nat Rev Neurosci 7:268-277. CrossRef Medline

Anderson NE, Kiehl KA (2012) The psychopath magnetized: insights from brain imaging. Trends Cogn Sci 16:52-60. CrossRef Medline

Apps MA, Ramnani N (2014) The anterior cingulate gyrus signals the net value of others' rewards. J Neurosci 34:6190-6200. CrossRef Medline

Apps MA, Green R, Ramnani N (2013a) Reinforcement learning signals in the anterior cingulate cortex code for others' false beliefs. Neuroimage 64:1-9. CrossRef Medline

Apps MA, Lockwood PL, Balsters JH (2013b) The role of the midcingulate cortex in monitoring others' decisions. Front Neurosci 7:251. Medline

Apps MA, Lesage E, Ramnani N (2015) Vicarious reinforcement learning signals when instructing others. J Neurosci 35:2904-2913. CrossRef Medline

Ashburner J, Friston KJ (2005) Unified segmentation. Neuroimage 26:839851. CrossRef Medline

Barbas H, Ghashghaei H, Dombrowski SM, Rempel-Clower NL (1999) Medial prefrontal cortices are unified by common connections with superior temporal cortices and distinguished by input from memory-related areas in the rhesus monkey. J Comp Neurol 410:343-367. CrossRef Medline

Behrens TE, Hunt LT, Woolrich MW, Rushworth MF (2008) Associative learning of social value. Nature 456:245-249. CrossRef Medline

Bird G, Viding E (2014) The self to other model of empathy: Providing a new framework for understanding empathy impairments in psychopathy, autism, and alexithymia. Neurosci Biobehav Rev 47:520-532. CrossRef Medline

Blair RJ (2005) Responding to the emotions of others: dissociating forms of empathy through the study of typical and psychiatric populations. Conscious Cogn 14:698-718. CrossRef Medline

Boorman ED, O'Doherty JP, Adolphs R, Rangel A (2013) The behavioral and neural mechanisms underlying the tracking of expertise. Neuron 80:1558-1571. CrossRef Medline

Braams BR, Güroğlu B, de Water E, Meuwese R, Koolschijn PC, Peper JS, Crone EA (2014) Reward-related neural responses are dependent on the beneficiary. Soc Cogn Affect Neurosci 9:1030-1037. CrossRef Medline

Brazil IA, Mars RB, Bulten BH, Buitelaar JK, Verkes RJ, De Bruijn ER (2011) A neurophysiological dissociation between monitoring one's own and others' actions in psychopathy. Biol Psychiatry 69:693-699. CrossRef Medline

Brett M, Anton J-L, Valabregue R, Poline J-B (2002) Region of interest analysis using the MarsBar toolbox for SPM 99. Neuroimage 16:S497.

Chang SW, Gariépy JF, Platt ML (2013) Neuronal reference frames for social decisions in primate frontal cortex. Nat Neurosci 16:243-250. Medline

de Waal FB (2008) Putting the altruism back into altruism: the evolution of empathy. Annu Rev Psychol 59:279-300. CrossRef Medline

Deichmann R, Gottfried JA, Hutton C, Turner R (2003) Optimized EPI for fMRI studies of the orbitofrontal cortex. Neuroimage 19:430-441. CrossRef Medline

Delmonte S, Gallagher L, O'Hanlon E, McGrath J, Balsters JH (2013) Functional and structural connectivity of frontostriatal circuitry in autism spectrum disorder. Front Hum Neurosci 430.

Engen HG, Singer T (2013) Empathy circuits. Curr Opin Neurobiol 23: 275-282. CrossRef Medline

Fareri DS, Niznikiewicz MA, Lee VK, Delgado MR (2012) Social network modulation of reward-related signals. J Neurosci 32:9045-9052. CrossRef Medline

Gabay AS, Radua J, Kempton MJ, Mehta MA (2014) The Ultimatum Game and the brain: A meta-analysis of neuroimaging studies. Neurosci Biobehav Rev 47:549-558. CrossRef Medline

Haber SN, Kunishio K, Mizobuchi M, Lynd-Balta E (1995) The orbital and medial prefrontal circuit through the primate basal ganglia. J Neurosci 15:4851-4867. Medline
Haroush K, Williams ZM (2015) Neuronal prediction of opponent's behavior during cooperative social interchange in primates. Cell:1-13.

Hillman KL, Bilkey DK (2012) Neural encoding of competitive effort in the anterior cingulate cortex. Nat Neurosci 15:1290-1297. CrossRef Medline

Jones RM, Somerville LH, Li J, Ruberry EJ, Libby V, Glover G, Voss HU, Ballon DJ, Casey BJ (2011) Behavioral and neural properties of social reinforcement learning. J Neurosci 31:13039-13045. CrossRef Medline

Lamm C, Decety J, Singer T (2011) Meta-analytic evidence for common and distinct neural networks associated with directly experienced pain and empathy for pain. Neuroimage 54:2492-2502. CrossRef Medline

Lockwood PL, Bird G, Bridge M, Viding E (2013a) Dissecting empathy: high levels of psychopathic and autistic traits are characterised by difficulties in different social information processing domains Front Hum Neurosci 7:760.

Lockwood PL, Sebastian CL, McCrory EJ, Hyde ZH, Gu X, De Brito SA, Viding E (2013b) Association of callous traits with reduced neural response to others' pain in children with conduct problems. Curr Biol 23: 901-905. CrossRef Medline

Lynd-Balta E, Haber SN (1994) Primate striatonigral projections-a comparison of the sensorimotor-related striatum and the ventral striatum. J Comp Neurol 345:562-578. CrossRef Medline

Markowitsch HJ, Emmans D, Irle E, Streicher M, Preilowski B (1985) Cortical and subcortical afferent connections of the primates temporal pole-a study of rhesus-monkeys, squirrel-monkeys, and marmosets. J Comp Neurol 242:425-458. CrossRef Medline

Mobbs D, Yu R, Meyer M, Passamonti L, Seymour B, Calder AJ, Schweizer S, Frith CD, Dalgleish T (2009) A key role for similarity in vicarious reward. Science 324:900. CrossRef Medline

Morelli SA, Sacchet MD, Zaki J (2015) Common and distinct neural correlates of personal and vicarious reward: a quantitative meta-analysis. Neuroimage 112:244-253. CrossRef Medline

Reniers RL, Corcoran R, Drake R, Shryane NM, Völlm BA (2011) The QCAE: a questionnaire of cognitive and affective empathy. J Pers Assess 93:84-95. CrossRef Medline

Rilling JK, Sanfey AG (2011) The neuroscience of social decision-making. Annu Rev Psychol 62:23-48. CrossRef Medline

Rogers RD, Ramnani N, Mackay C, Wilson JL, Jezzard P, Carter CS, Smith SM (2004) Distinct portions of anterior cingulate cortex and medial prefrontal cortex are activated by reward processing in separable phases of decision-making cognition. Biol Psychiatry 55:594-602. CrossRef Medline

Rudebeck PH, Buckley MJ, Walton ME, Rushworth MF (2006) A role for the macaque anterior cingulate gyrus in social valuation. Science 313: 1310-1312. CrossRef Medline

Ruff CC, Fehr E (2014) The neurobiology of rewards and values in social decision making. Nat Rev Neurosci 15:549-562. CrossRef Medline

Sallet J, Quilodran R, Rothé M, Vezoli J, Joseph JP, Procyk E (2007) Expectations, gains, and losses in the anterior cingulate cortex. Cogn Affect Behav Neurosci 7:327-336. CrossRef Medline

Schultz W (2013) Updating dopamine reward signals. Curr Opin Neurobiol 23:229-238. CrossRef Medline

Seltzer B, Pandya DN (1989) Frontal-lobe connections of the superior temporal sulcus in the rhesus-monkey. J Comp Neurol 281:97-113. CrossRef Medline

Shidara M, Richmond BJ (2002) Anterior cingulate: Single neuronal signals related to degree of reward expectancy. Science 296:1709-1711. CrossRef Medline

Simms ML, Kemper TL, Timbie CM, Bauman ML, Blatt GJ (2009) The anterior cingulate cortex in autism: heterogeneity of qualitative and quantitative cytoarchitectonic features suggests possible subgroups. Acta Neuropathol 118:673-684. CrossRef Medline

Singer T, Lamm C (2009) The social neuroscience of empathy. Ann N Y Acad Sci 1156:81-96. CrossRef Medline

Vogt BA, Nimchinsky EA, Vogt LJ, Hof PR (1995) Human cingulate cortexsurface-features, flat maps and cytoarchitecture. J Comp Neurol 359: 490-506. CrossRef Medline

Yeterian EH, Pandya DN (1991) Prefrontostriatal connections in relation to cortical architectonic organization in rhesus-monkeys. J Comp Neurol 312:43-67. CrossRef Medline 